\title{
SISTEMA DE GESTIÓN EN SALUD OCUPACIONAL: RETO PARA LOS ADMINISTRADORES DEL SECTOR SALUD EN CÓRDOBA - COLOMBIA*
}

\author{
MÓNICA I. HANNA LAVALLE**
}

Recibido 10 de Septiembre de 2012 / Enviado para Modificación 13 de Octubre de 2012 / Aceptado 20 de Noviembre de 2012

\begin{abstract}
RESUMEN
La presente investigación tiene como objetivo evaluar los sistemas de gestión en seguridad y salud ocupacional (SGSO) en Empresas Sociales del Estado de mediana complejidad en Córdoba (Col). Para ello se realiza un estudio descriptivo, transversal, con enfoque cuantitativo. La obtención de los datos se realizó teniendo en cuenta los requerimientos de la norma OHSAS 18001 y la normatividad vigente, estos fueron procesados con el programa estadístico SPSS versión 20. Los resultados evidencian que los administradores no invierten en el Sistema Integral de Gestión en Seguridad y Salud Ocupacional, estos sistemas continúan en etapa inicial, no existe asociación de los elementos que lo conforman y presentan deficiencias. Además, el capital humano no posee una cultura preventiva en seguridad laboral, todo lo anterior va en contra de las politicas públicas que rigen este Sistema.
\end{abstract}

Palabras claves: sistemas, gestión, salud ocupacional, administración, politica pública, regulación, capital humano.

\begin{abstract}
This research aims to evaluate the safety management systems and occupational health (SGSSO) in State Social Enterprises of medium complexity in Cordoba (Col). This is a descriptive study, cross with a quantitative approach. The data collection was performed taking into account the requirements of OHSAS 18001 and the regulations in force; these were processed using SPSS version 20. The

\footnotetext{
* Toda la información aquí suministrada es veraz, recolectada con instrumento y con el consentimiento informado según Resolución 008430 del Consejo Nacional de Salud (Ítem IV), firmado por los coordinadores del programa de Salud ocupacional de las instituciones del estudio.

** Enfermera, Candidata a Magister de Administración de la Universidad Nacional de Colombia. Administradora en salud y especialista en salud ocupacional. Docente de la Universidad de Córdoba, Facultad de Ciencias de la Salud, Departamento de Enfermería. Correo Electrónico: monikmayi@hotmail.com.
} 
results show that managers do not invest in the Integrated Management System Occupational Health and Safety, these systems are still in early stage, there is no association of the elements that comprise it and deficiencies. In addition, human capital does not have a culture of prevention in occupational safety, the above goes against public policies governing this system.

Key words: systems, management, occupational health, administration, public policy, regulation, human capital.

Classification Journal Economic Literature (JEL): J24, M21, M51, L38.

\section{INTRODUCCIÓN}

Las instituciones de salud se convirtieron en Empresas Sociales del Estado, estas entidades públicas deben concurrir a la financiación de los servicios de salud con recursos propios y fiscales enunciados por el gobierno (1). De allí la necesidad que inicien un proceso competitivo, no olvidando la importancia de la satisfacción de los clientes internos y externos (2).

Para lograr lo anterior, el capital humano debe conocer normas o conceptos que permiten el buen desarrollo del clima organizacional, la actividad laboral y el cumplimiento de las políticas públicas. Los funcionarios del sector salud del área administrativa y asistencial deben basarse en la estandarización de actividades de la organización (3).

Existen requerimientos que permiten evaluar los sistemas, entre ellos el Sistema de Gestión de la Salud y la Seguridad en el trabajo reconocido internacionalmente por la norma (4).

Para la conformación del Sistema de Gestión de Seguridad y Salud Ocupacional, el administrador se debe apoyar en el Decreto 614,1984 (5), la Resolución 1016 de 1989 (6), el Sistema General de Riesgos Profesionales (SGRP); este último creado mediante la expedición del tercer libro de la Ley 100/93 y reglamentado por el Decreto 1295/94 (7) y la ley 1562 del 2012 (8), por la cual se modifica el Sistema de Riesgos Laborales.

Con el cumplimiento de las normas y la legislación, debería existir una cultura organizacional y un clima empresarial donde la Salud Ocupacional evitaría accidentes de trabajo y enfermedades profesionales, pero las estadísticas demuestran lo contrario. El principal propósito de las normas consiste en mejorar el desempeño, la productividad en la 
prestación de servicios que respondan a las necesidades y expectativas de sus clientes. (9), estas normas se consideran complementarias entre ellas y su aplicación una inversión para la institución.

Si bien este no es solamente un problema colombiano, en la actualidad en el mundo la accidentalidad y las enfermedades profesionales que cada año se producen muestran estadísticas de 2.2 millones de fallecimientos relacionados con el trabajo.

La mayor cuota de victimas la aportan las enfermedades profesionales con 1.7 y 2 millones de fallecimientos por año. Así mismo, en América Latina ocurren 5 millones de accidentes cada año, 17.500 accidentes diarios, con el fallecimiento de 300 trabajadores $y$, finalmente, 38 accidentes por minuto, lo cual genera, además de los efectos humanos, pérdidas millonarias a empresarios (10).

En Colombia las estadísticas del año 2010, muestran un total de 163.408 presuntos accidentes de trabajo sucedidos y calificados 148.250, además de 2016 enfermedades profesionales calificadas (11).

En el Departamento de Córdoba las cifras indican 1.594 accidentes de trabajo reportados; esta cifra se debe a la poca información existente sobre riesgos laborales, accidentalidad y enfermedades ocupacionales; en muchas ocasiones son reportados como morbilidad dentro del Sistema General de Seguridad Social en Socia 1 (SGSSS).

Esta última afirmación es ratificada por la Oficina Internacional del Trabajo (12) donde se trató el tema "los desafíos y propuestas en seguridad y salud en el trabajo, insumo para el foro del empleo", llegándose a la siguiente conclusión:

“... Se sugiere involucrar al sector de la salud de manera más concreta en este esfuerzo, para complementar las acciones desarrolladas en el sector laboral, en el reconocimiento de patologías profesionales.

Adicionalmente, teniendo en cuenta las dificultades que se presentan en la recolección de datos estadísticos, es necesario poner en marcha mecanismos adecuados en sistematización y recolección de datos sobre accidentes de trabajo y enfermedades profesionales."(13). Una vez el sector salud tenga claro lo anterior se podrá convertir esta acción en una fortaleza interna y externa de las empresas. 
Las empresas Sociales del Estado disponen para regular las actividades de esta área de una persona encargada que programe y articule las actividades con la Administradora de Riesgos Profesionales, escogida por el director de la institución.

Se aclara que el empleador tiene la obligación de cumplir con la legislación en materia de Seguridad y Salud en el trabajo, y tiene el deber de organizar la gestión de los sistemas de Salud Ocupacional en cuanto a las medidas de prevención de accidentes y enfermedades del trabajo en la empresa. (14).

Las debilidades de este sistema pueden ser causa de disminución de la productividad individual y de la calidad de vida de los trabajadores, de allí la importancia de evaluarlos. Este trabajo aportaría a las ciencias de gestión información valiosa, para determinar dónde se encuentran los errores del sistema de gestión en Seguridad y Salud ocupacional de las Empresas Sociales del Estado.

Como bien dice Morin: “... Hay problemas de la verdad en relación a las finalidades y las normas, y esto pone en cuestión las elecciones fundamentales: tenemos que saber qué hacemos estas elecciones, pero debemos saber también, en ese momento, que la verdad normativa, ética, política, no es de la misma naturaleza que la de aquella que constata que una mesa es una mesa" (15).

Lo anterior lleva a deducir que el administrador del sector salud debe tener conocimiento, para desarrollar e implementar la política de seguridad y salud ocupacional, gestionando los riesgos laborales.

Además, debe tener en cuenta la norma Ocupacional OHSAS 18001(10), ISO 14001:2004, a fin de establecer el sistema Integral de Gestión de la Calidad, NTCGP 1000 norma Técnica de Calidad en Gestión Pública, Decreto 614 de 1984, y la resolución 1016 de 1989, ya antes mencionados, enfatizando estos últimos en proteger al trabajador en su ambiente laboral, contribuyendo así al bienestar físico, mental y a su confort.

\section{MARCO REFERENCIAL}

Los SGSSO son la parte del sistema gerencial que facilita la administración de los riesgos de la salud y la seguridad, generados por la actividad laboral. Esto incluye la estructura organizacional, la planeación, prácticas, procesos, procedimientos y logros para el desarrollo, implementación y mantenimiento de la política de seguridad y salud ocupacional. (16) 
Estos deben ser liderados por la gerencia de las empresas e instituciones de los diferentes sectores de la economía, ya que se consideran una ayuda para mejorar la calidad de los servicios, productos y el medio ambiente laboral. Su importancia radica en la interdisciplinariedad, esta permite contener diversos campos especializados, lo que logra un entorno adaptado a las necesidades físicas y mentales de los trabajadores, cumplir con la legislación, reducir costos al manejar la seguridad y realizar marketing mostrando una buena imagen empresarial (17).

\section{ANTECEDENTES DE MODELOS DE GESTIÓN EN S Y SO}

Con el fin de realizar acuerdos de libre comercio, se obliga la creación de un modelo estándar de gestión para la prevención de los diferentes riesgos en las áreas laborales. Para ello, la International Organization for standarización (ISO), invitóa esteproceso a sindicatos, aseguradores, gobiernos y trabajadores conformados por 45 países, estos dieron aportes a esta normatividad. De allí surgen numerosas ideas, que lograron concretarse y servir de aporte para incentivar a otras organizaciones a crear su propio modelo.

En Colombia el Instituto Colombiano de Normas Técnicas y Certificación (ICONTEC), es el organismo nacional encargado de la regulación y normatización, según el decreto 2269 de 1993, entidad que brinda soporte y desarrollo al productor y protección al consumidor. Esta institución mediante consejo directivo del 2007 ratifica a las OHSAS 18001 como una norma reconocida, como guía para la implementación, evaluación y certificación de los S y SO. (18).

Para efectos de este estudio se escoge la Norma OHSAS 18001, la cual se considera una guía para la evaluación del S y SO por tener las siguientes características:

Esta norma es desarrollada para ser compatible con ISO 9001:1994 e ISO: 14001:1996, posee un mayor grado de exigencia, demanda desarrollo y mantenimiento de procedimientos, trata de actividades preventivas y se encuentra acorde con mecanismos para retroalimentación de la gestión.

\section{NORMA OHSAS 18001}

La comunidad internacional decidió orientar un modelo que permite armonizar un estándar de los requisitos en seguridad y salud ocupacional, por lo cual impulsa la implementación del modelo BS OHSAS 18001 (Occupational Health and Safety Assessment, Series) (19). 
Este sistema busca: eliminar o minimizar los riesgos asociados con las actividades empresariales; implementar, mantener y mejorar en forma continua un sistema de gestión; asegurar la política empresarial, demostrar conformidad con la norma, buscando la certificación o registro del sistema de gestión de seguridad y salud ocupacional, mediante una empresa externa reconocida.

Para el funcionamiento del sistema se debe tener en cuenta las siguientes recomendaciones: reconocimiento de la necesidad de integrar los sistemas de gestión, compromiso, existencia de un enfoque de sistema en la gestión empresarial, enfoque de procesos, orientación hacia la mejora continua (20), basado en el Ciclo de DEMING.

\section{ANTECEDENTES INVESTIGATIVOS}

La Oficina Internacional del Trabajo (OIT) muestra inquietud por la situación laboral, las condiciones de seguridad y salud ocupacional en muchos países. En razón a lo anterior, se encuentran los siguientes referentes:

Investigaciones que afirman que la mayoría de las empresas en países como Haití, Panamá, República Dominicana, entre otros, no cuentan con sistemas de gestión eficiente y eficaces; trayendo como consecuencia irregularidades dentro de los sitios de trabajo; el documento titulado “Desafíos y propuestas en seguridad y salud en el trabajo, insumos para el foro de empleo, es muestra de una de ellas (21).

En España, analizaron los riesgos en centros sanitarios de ese país, con el fin de identificar las principales concepciones teóricas que sustentan un modelo para la mejora continua de la gestión de la Seguridad e Higiene Ocupacional (SHO) (22).

Países como Cuba evaluaron un sistema de gestión de la seguridad e higiene ocupacional, donde demuestran el diseño de un sistema de indicadores de desempeño, desde la perspectiva de la eficiencia, eficacia y efectividad (23).

Entre las investigaciones institucionales relacionadas con este tema, se encuentra la realizada en Colombia por el Consejo Colombiano de Seguridad, titulada "Evaluación de la gestión del sistema de seguridad, salud ocupacional y ambiente para contratistas", cuyo fin, es la disminución de la accidentalidad y necesidad de controlar los riesgos, 
dando a sus procesos y a sus contratistas confiabilidad en la ejecución del servicio. Otra demostración, son los aportes de "Bogotá emprende", quienes realizaron una presentación denominada "Conoce los requisitos de calidad que exige el mercado", basados en los contenidos de cómo implementar un sistema de gestión en una empresa (24).

Se enuncia además los aportes de Coello, Y. Con el trabajo titulado "Diseño del Sistema de Gestión Integrado Calidad-Medio Ambiente-Seguridad y Salud en el Trabajo, en el Proceso Ejecución de Inversiones de la Empresa Eléctrica Holguín". Peña, D.; Rigol, B. y Moreno, M., quienes afirman, que un Sistema Integrado de Gestión (SIG) puede cubrir todos los aspectos de la gestión institucional (25).

\section{MATERIALES Y MÉTODOS}

Estudio descriptivo, transversal con enfoque cuantitativo. Muestreo por conveniencia en 4 instituciones de mediana complejidad del departamento de Córdoba. Se recolectó la información a través de encuestas a los coordinadores de área, se determinan las condiciones del sistema de seguridad y salud ocupacional, mediante una lista de chequeo basada en la norma OHSAS 18001 y la normatividad vigente.

Las variables medidas en la encuesta incluyen generalidades, consideraciones del sistema, verificación de requisitos legales, reporte de accidentes y enfermedades laborales y actividades de evaluación y control del sistema.

Como medida de fiabilidad de ambos instrumentos, fue utilizada el Alfa de Cronbach (26). Los hallazgos encontrados en la medida se obtienen, cuando los ítems discriminan si las respuestas son muy parecidas (todos de acuerdo, o en desacuerdo), la varianza de los ítems es baja y también la fiabilidad.

En este caso, la medida de fiabilidad muestra una buena consistencia interna, ya que el coeficiente resulta ser 0.7. Establecer la consistencia interna de una escala es una aproximación a la validación del constructo y consiste en la cuantificación de la correlación que existe entre los ítems que la componen.

Por otra parte existen diferentes coeficientes como el método de Rulon (1939), el coeficiente de Kristof (1974), el coeficiente de Angoff-Feldt (1975), el coeficiente beta, propuesto por Raju (1977), teniendo en cuenta 
las múltiples escalas utilizadas el coeficiente alfa de Cronbach es de gran utilidad por sus propiedades. Su popularidad se debe a la practicidad de su uso, ya que requiere una sola administración de la prueba $(27,28,29,30)$.

\section{Análisis Estadístico:}

Se analiza la información con el paquete estadístico SPSS versión 20 (31), con análisis multivariado, en este se establecen relaciones entre variables. Obteniendo un plano factorial que las ilustra. Inicialmente se muestran los valores propios y porcentajes de inercia, que indican el número de ejes que se toman para formar los planos factoriales.

En este caso solo con el primer plano se considera significativo. Luego se presentan las coordenadas factoriales que indican las ubicación de los puntos en el plano, se presentan las contribuciones de los puntos a los ejes, finalmente los cosenos y cuadrados que representan la importancia del punto hacia el eje; también los valores de los test para evaluar la significancia de los puntos en la gráfica (Ver gráfico 1.)

\section{RESULTADOS}

Las 4 empresas sociales del estado objeto de estudio son contratistas, con el fin de brindar la atención directa en salud de 875.600 usuarios del régimen subsidiado, además prestan servicio a la población en situación de pobreza y vulnerabilidad. Estas instituciones poseen un total de 1.550 trabajadores de los cuales 258 son de planta y 1.292 de contrato (32).

El análisis de resultado demostró que: las políticas de seguridad y salud ocupacional, son deficientes, el apoyo de las directivas a las actividades de esta área es de un 50\%, solo dos directivos muestran interés. Las administraciones no tienen como relevante el Sistema de gestión en salud ocupacional, enfocan su gestión hacia la producción, aumento de la rentabilidad y disminución de costos en procesos o procedimientos.

El 100\% no asigna rubro presupuestal para el sistema, en un 75\% de los casos no existe el cargo de coordinador, pero esta función la realizan en este mismo porcentaje de los casos profesionales administrativos que no poseen el perfil específico. En su mayoría son mujeres con edad promedio de 44 años, vinculados de planta. El 100\% no utiliza totalmente el ciclo de DEMING (planear, Hacer, Verificar y actuar) (Ver Gráfico 2). 
Las políticas de seguridad y salud ocupacional establecidas son las exigidas en la norma, los objetivos del sistema existen, pero no se cumplen a cabalidad. Hay desconocimiento de la normatividad de sistemas de gestión en dos de las instituciones, a pesar de que se realizan planes de capacitación en coordinación con las administradoras de riesgos laborales.

Los coordinadores afirman no tener disponibilidad presupuestal, se identificaron falencias del $100 \%$ en el proceso de comunicación, el $75 \%$ no realiza inducción a sus trabajadores en forma general, sobre todo a subcontratistas: en especial a personal de servicios generales y clientes externos. La cultura preventiva es aplicada por un $40 \%$ de los funcionarios, no se realizan seguimientos de accidentes de trabajo y no se aplican medidas preventivas en un $75 \%$.

Las instituciones cuentan con la documentación exigida por el sistema en un $45 \%$, la cual corresponde en su gran mayoría al programa de salud ocupacional. Los comités paritarios de salud ocupacional se encuentran constituidos y activos en un $100 \%$, pero no se les asigna a los miembros del comité el tiempo requerido para sus funciones. La lista de chequeo de la norma, no demuestra la existencia de un sistema interrelacionado, los elementos de este funcionan separadamente.

El 50\% de las instituciones tienen planes de acción, panorama de factores de riesgos y reportes de accidentabilidad laboral. Pero no existen controles suficientes para evitar los riesgos identificados, las auditorias, la vigilancia y control se ejerce en un $25 \%$. El $75 \%$ de las empresas estudiadas no evalúan su plan de mejoramiento anterior. Ver (Gráfica 3.)

\section{DISCUSIÓN}

"La gestión es una continuidad de acciones cuya aplicabilidad está relacionada con el (ciclo de Deming). Lo anterior exigido por la normatividad de gestión pública en empresas del Estado colombiano (33). Las instituciones de salud del Estado en el Departamento de Córdoba, tratan de aplicar este ciclo, pero en realidad utilizan el modelo del primer nivel de integración: donde la norma, es solo un requisito para la supervisión de entes reguladores, olvidando en primera instancia que la seguridad laboral es un servicio de carácter obligatorio e irrenunciable de los trabajadores (34).

Los Planes de trabajo anual y la financiación de los Programas de Salud Ocupacional deben realizarlo los empleadores, quiénes están obligados 
a proporcionar, cuatro horas semanales dentro de la jornada normal de trabajo a cada miembro del comité paritario de salud ocupacional para su funcionamiento, lo cual según información recolectada no se cumple a cabalidad (35).

Entre las exigencias actuales, el administrador debe nombrar un funcionario que se encargue de "Administrar el riesgo", con el fin de mantener la estabilidad financiera de la empresa, proteger activos e ingresos $\mathrm{y}$, minimizar pérdidas ocasionadas por la ocurrencia de dichos riesgos (36).

En consecuencia la administración del sector salud en Córdoba, no logra espacios de seguridad laboral. Se evidencia además que existe carencia de conocimiento sobre las ciencias del comportamiento organizacional por parte de las directivas, lo que obliga a citar a Omar Aktuf quién afirma que:

"Todo sistema es de gran componente ideológico, la administración está condenada a encarar frontalmente la explicación, legitimación y la auto justificación que realiza un detrimento de la concordancia real y operacional" (37).

Es necesario entonces que los administradores de las empresas sociales del estado en el Departamento de Córdoba, logren concadenar lo normativo, lo real y lo operacional como una sola acción institucional.

\section{CONCLUSIONES}

La alta dirección debe definir y autorizar la política de S y SO (seguridad y salud ocupacional) de la organización, asegurar que dentro del alcance definido de su sistema de gestión se apropie la naturaleza y escala de los riesgos de la organización. Es evidente que existe un problema administrativo, además de un deficiente autocuidado de las personas que irónicamente propenden por la salud de los demás.

Por lo tanto es de suma importancia antes que documentar e implementar normas, crear conciencia en cada uno de los participantes del macro y micro sistema de seguridad y salud ocupacional.

\section{FINANCIACIÓN}

Esta investigación fue financiada con recursos propios del investigador principal. 


\section{INTERÉS DE CONFLICTOS}

El presente estudio se realizó con el fin de optar el título de Magister en Administración de la Facultad de Ciencias Económicas de la Universidad Nacional de Colombia.

\section{AGRADECIMIENTOS}

A los funcionarios de las instituciones del estudio, a las docentes de la Universidad de Córdoba Concepción Amador y Marilyn Villadiego, por su apoyo.

Sinceros agradecimientos a las instituciones de salud de esta investigación y sus administrativos por toda su colaboración, al estudiante de enfermería David Machado por su asistencia en la recolección de datos, a la universidad de Córdoba por su apoyo en el proceso de cualificación docente.

\section{REFERENCIAS BIBLIOGRÁFICAS}

1. Ministerio de Salud. La reforma a la seguridad social en salud. Antecedentes y resultados, acto de 1993, pub. 1-132. Santa Fe de Bogotá, Colombia. (1993).

2. Mora, D."La integración de los sistemas de gestión, un reto para la empresa cubana contemporánea. Caso de estudio" en Observatorio de la Economía Latinoamericana, No 121, 2009.

3. Sistema de Gestión de la Calidad (ISO-9000), 1987. Organización Internacional de Normalización, Ginebra, Suiza.

4. Normas OHSAS 18001:2007), Consejo Colombiano de Seguridad, Sistemas de Gestión en Seguridad y Salud ocupacional, Bogotá, Colombia.

5. Ministerio de Salud. decreto 614 de (1984) Marzo 14. Diario oficial del estado 36561.Bases para organización y administración en salud ocupacional. Santa fe de Bogotá, Colombia.

6. Ministerio de protección Social, (1989). Resolución 1016, por el cual se reglamenta la organización, funcionamiento y forma de los Programas de Salud Ocupacional. Bogotá, Colombia.

7. Ministerio de salud. Decreto 1295. (1.994). Determina la organización y administración del Sistema General de Riesgos Profesionales. Bogotá, Colombia. 
8. Ministerio de salud. Decreto 1562. (2012). Modificaciones al sistema general de riesgos profesionales. Bogotá, Colombia.

9. Modelo estándar de Control Interno para el estado Colombiano. Sistema de gestión pública. Bogotá, Colombia (2005).

10. Organización Internacional del Trabajo. (2005). Informe de la OIT sobre muertes laborales Comunicación de Responsabilidad Social de la Empresa, Buenos Aires (Argentina).

11. Federación de Aseguradores Colombianos. (2010). Informe anual por reportes de accidentes de trabajo y enfermedades profesionales, [serial on line]. Septim-Novim [citado 2011 No 15];1(1):[28] desde :URL: http:/ / www.fasecolda.com/fasecolda/

12. Organización Internacional del Trabajo (2005). Desafíos y propuestas en seguridad y salud en el trabajo, insumos para el foro de empleo Oficina Subregional para Centroamérica, Hatí, Panamá. Oficina Subregional para Centroamérica, Haití, Panamá y República Dominicana.

13. Organización Internacional del Trabajo. Registro y notificación de accidentes del trabajo y enfermedades profesionales, Repertorio de recomendaciones prácticas de la OIT. Ginebra. 2002. P.3.

14. Instituto Salud y trabajo, Informe general presentado a la OIT, 2011. Sistematización y recolección de datos sobre ATEP.pág.13.

15. Morin E. (1999). El método. La naturaleza de la naturaleza, volumen I Ed. Cátedra, Madrid, España. pág 108

16. BS 8800. Guide to Occupational health and safety Management Systems (1996).

17. Lázaro, L. Prevención de fatalidades a través del análisis de peligros operacionales. Tesis de la Universidad Católica, Perú (2007).pág.10.

18. Norma Técnica Colombiana. OHSAS18001.http://comunica.sena. edu.co/sigc/docus/ntc_ohsas_18001_2007.pdf [fecha de consulta: 25 de Septiembre del 2012].

19. Occupational Health and Safety Assessment Series (2007). Sistema de Gestión de la Calidad. Normas OHSAS 18001, Consejo Colombiano de Seguridad, Sistemas de Gestión en Seguridad y Salud ocupacional. Santa fe de Bogotá, Colombia.

20. Morales, D. (2009). “La integración de los sistemas de gestión, un reto para la empresa cubana contemporánea. Caso de estudio" en Observatorio de la Economía Latinoamericana. 
21. Forastieri, V. Desafíos y propuestas en seguridad y salud en el trabajo, insumos $\mathrm{p}$ ara el foro de empleo. Organización Internacional del Trabajo, (2005), Haití.

22. Atence, J. Análisis de Riesgos en Centros Sanitarios. Aspectos Metodológicos. Revista Medicina del Trabajo. Tomo XLIV. Nr. 174. (1997), España.

23. ÁLVAREZ, L. PACHECO, A.. Guía para la instalación del Programa Permanente de Mejoramiento de la Productividad en las empresas cubanas. ISTH. Cuba: IPN-UPIICSA. (1993),México-Cuba

24. Bogotá Emprende. Documento Contenido matriz, plan de empresa, Octubre. (2006), Bogotá Emprende

25. COELLO, Y. Diseño del Sistema de Gestión Integrado Calidad-Medio Ambiente-Seguridad y Salud en el Trabajo, en el proceso Ejecución de Inversiones de la Empresa Eléctrica Holguín (2005).

26. Cronbach, L. Coefficient alpha and the internal structure of test. Psychometrika, (1951). 16:297-334.

27. Rulon PJ. A simplified procedure for determining the reliability of a test of split halves. Harvard Educ Rev. 1939;9:99- 103.

28. Kristof $\mathrm{W}$. Estimation of reliability and true score variance from a split of a test into three arbitrary parts. Psychometrika. 1974;39:491-99.

29. Feldt LS. Estimation of the reliability of a test divided into two parts of unequal length. Psychometrika. 1975;40:557- 561.

30. Raju NS. A generalization of coefficient alpha. Psychometrika. 1977;42:549-65.

31. Pardo A, \& Ruiz M.A. Statistical Product and Service Solutions, Guía para el análisis de datos, ed.11 Madrid (ES): McGraw-Hill, 2002, p.3.

32. Oficinas de talento humano Empresa Social del Estado.. Informe de jefes de Oficina de Talento Humano de las cuatro instituciones de salud del Estado. (2012), Montería-Córdoba.

33. WALTON, Mary. El Método Deming en la práctica (1992). Norma, Bogotá.

34. Constitución política de Colombia. Seguridad Laboral, artículos 48, 49, 53.http://cmsstatic.colombiaaprende.edu.co/cache/binaries/ articles 186370_constitucion_politica.pdf?binary_rand=1416 [fecha de consulta: 24 de Mayo del 2012]. 
35. Ministerio de salud. Resolución 2013, Por la cual se reglamenta la organización y funcionamiento de los Comités de Medicina, Higiene y Seguridad Industrial en los lugares de trabajo (1986). http:/ / ley100.com/ res_2013de1986. Artículo 7 [Fecha de consulta: 24 de Mayo del 2012].

36. Ministerio de la protección social, Circular 02, reglamenta los planes y financiación de programas de salud ocupacional. 2002, Bogotá, Colombia.

37. Aktouf, Omar. La administración entre tradición y renovación. Ed.4 artes gráficas del Valle. Cali, Colombia. 2009, p. 235.

\section{ANEXOS}

Gráfico No. 1. Valores propios y porcentajes de inercia:

\begin{tabular}{|l|r|r|r|}
\hline & \multicolumn{1}{|c|}{ F1 } & \multicolumn{1}{|c|}{ F2 } & \multicolumn{1}{c|}{ F3 } \\
\hline Valor propio & 0,720 & 0,576 & 0,340 \\
\hline Inercia (\%) & 44,009 & 35,185 & 20,806 \\
\hline$\%$ acumulado & 44,009 & 79,194 & 100,000 \\
\hline Inercia ajustada & 0,479 & 0,284 & 0,075 \\
\hline Inercia ajustada (\%) & 53,292 & 31,640 & 8,382 \\
\hline \% acumulado & 53,292 & 84,933 & 93,315 \\
\hline
\end{tabular}

Scree plot

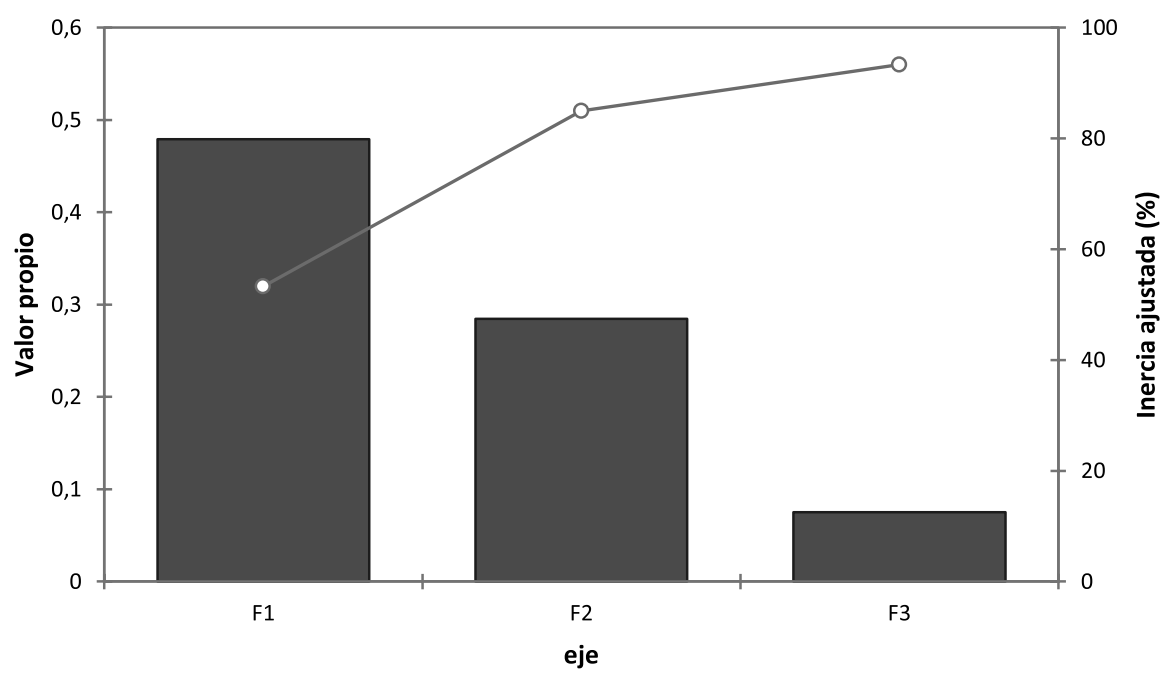

Coordenadas principales (Variables): 


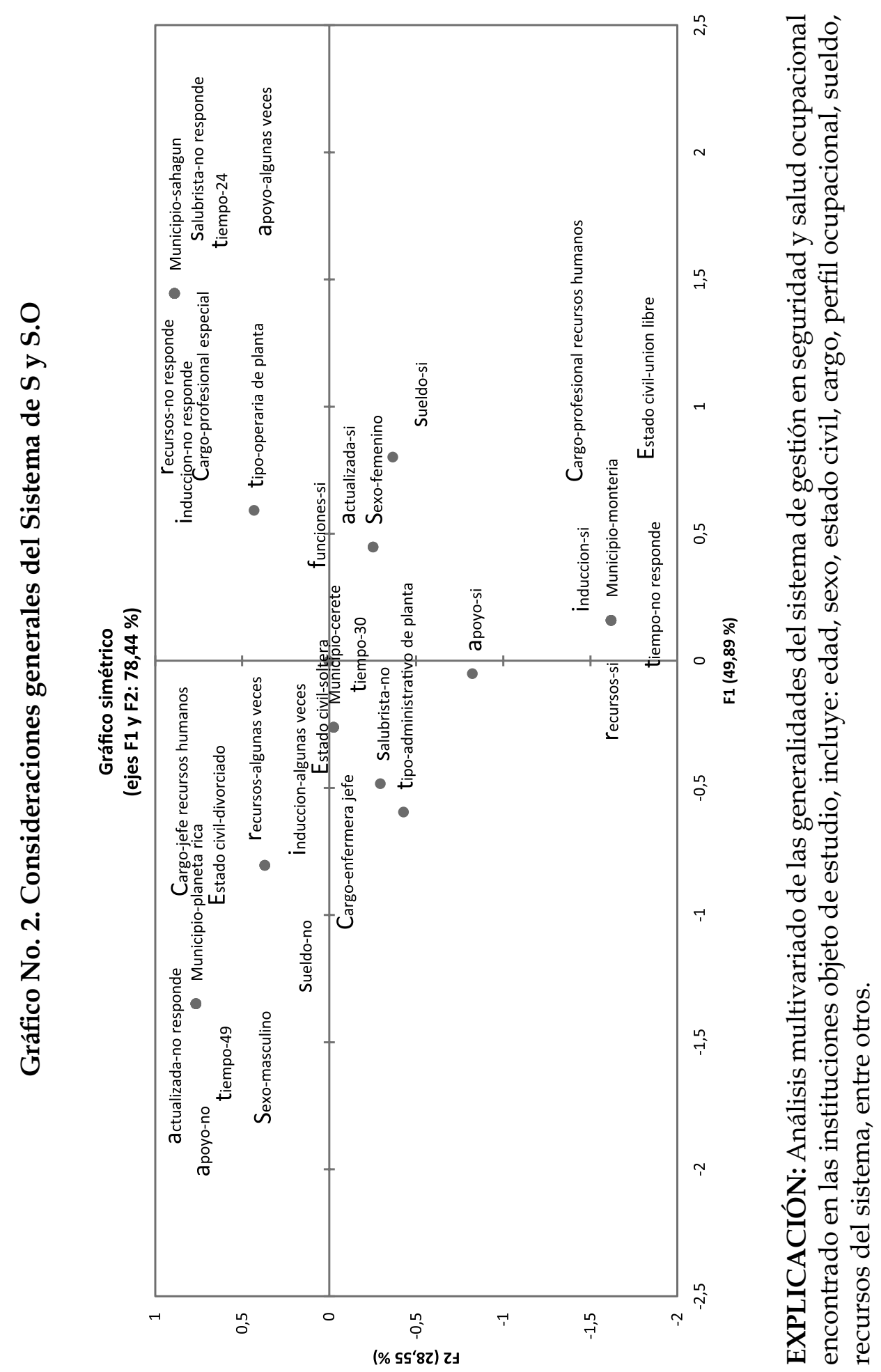




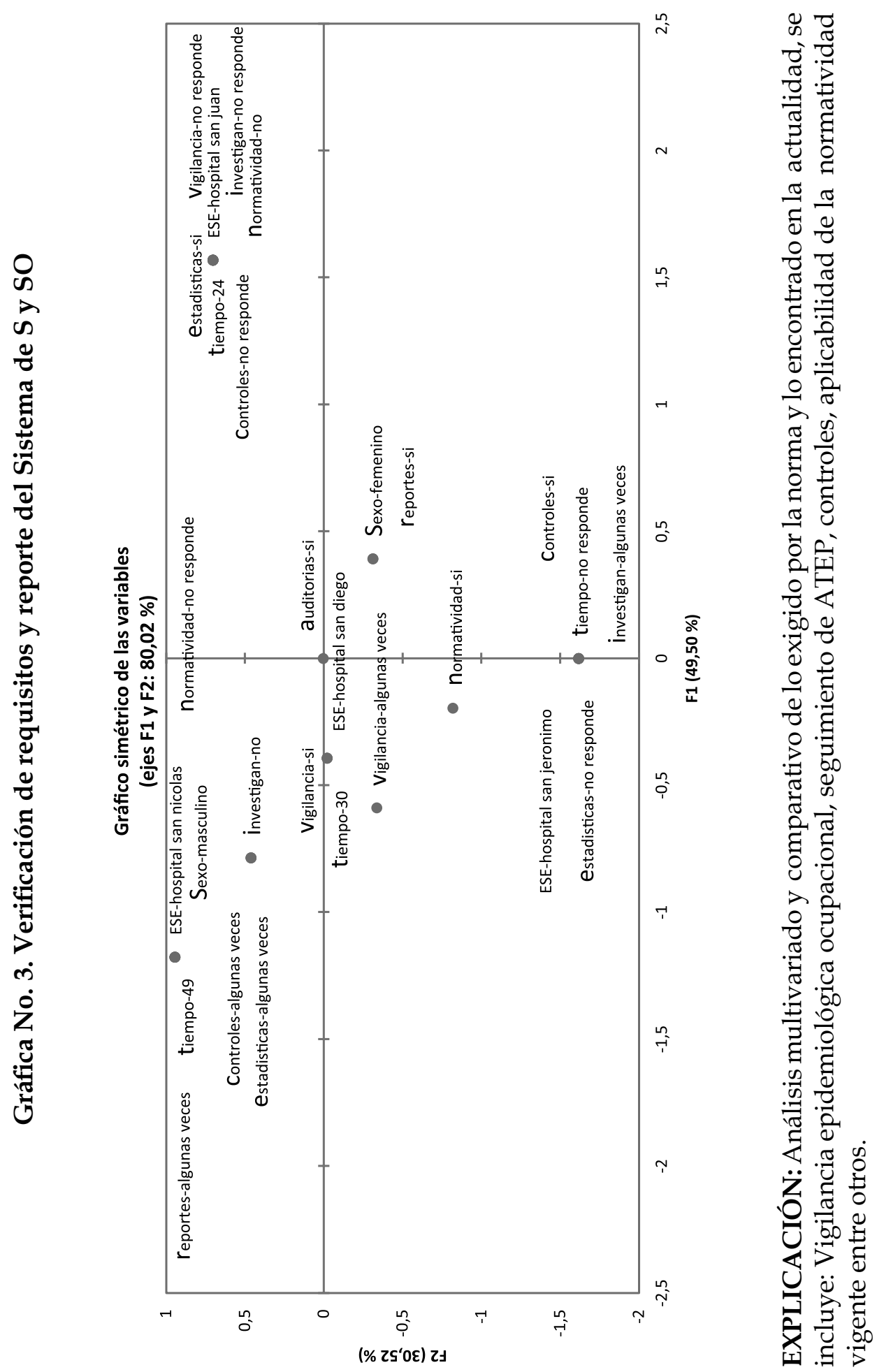

